\title{
Self-assembled extracellular matrix protein networks by microcontact printing
}

\author{
Nicoletta Sgarbi*, Dario Pisignano, Francesca Di Benedetto, Giuseppe Gigli, \\ Roberto Cingolani, Ross Rinaldi \\ NNL, National Nanotechnology Laboratory of Istituto Nazionale di Fisica della Materia (INFM), clo Dipartimento di Ingegneria \\ dell'Innovazione, Università di Lecce, via Arnesano, Lecce I-73100, Italy
}

Received 12 May 2003; accepted 11 August 2003

\begin{abstract}
Physiological patterns of the extracellular matrix protein, laminin-1, were obtained on glass substrates by physisorption-assisted microcontact printing. Besides the well-retained antigenicity confirmed by indirect immunofluorescence assays, we investigated the supramolecular organization of the proteins by atomic force microscopy. We found the characteristic protein self-assembling in polygonal networks with well-defined sub-100 nm quaternary structures of laminin. The formation of these physiological mesh-like protein matrices was obtained by means of one-step soft lithography without any preliminary functionalization of glass, which can be exploited for many possible applications for cell cultures and biomolecular devices.
\end{abstract}

(C) 2003 Elsevier Ltd. All rights reserved.

Keywords: Laminin; Micropatterning; Protein adsorption; Rapid prototyping; Atomic force microscopy

\section{Introduction}

In the last years bio-micropatterning techniques have attracted increasing interest due to the possibility to control the chemical surface properties of different types of substrates [1,2]. The capability of producing micrometer patterns of biological macromolecules has paramount importance for several applications, including advanced medical diagnostic, biological assays, miniaturized biosensors [3], hybrid molecular electronics [4] and rectifying devices [5,6]. In particular, soft lithography techniques $[7,8]$ are suitable to fabricate biomolecular patterns, by virtue of the wide range of usable target surfaces and solvents, including aqueous solutions, and of the very low operation cost, which makes them affordable for bio-organic laboratories. To date, a number of experiments employing imprinting processes [9], microfluidic networks [10-13], or microcontact printing $(\mu \mathrm{CP})$ [14-16] succeeded in achieving chemical contrast among different molecular monolayers, to

\footnotetext{
*Corresponding author. Tel.: + 39-08-3229-7283; fax: + 39-08-32326351.

E-mail address: nicoletta.sgarbi@unile.it (N. Sgarbi).
}

promote or prevent the deposition on specific areas of different elements, such as proteins or cells.

Concomitantly, substantial progress has been made in the understanding of the interactions between cells and their surroundings, and a fundamental role has been ascribed to the extracellular matrix (ECM) proteins, for their capability to directly affect the behaviour of the cells they interact with $[17,18]$. In vitro cell cultures should be able to recreate a physiological environment both from the topological and from the biochemical point of view, in order to simulate in vivo cellular functions. This demand is not fully satisfied by traditional cell cultures, which produce uniform distributions of ECM proteins and cells on the culture surface, and consequently do not allow to control the shape and the behaviour of the cells. This might result in morphological and functional differences with respect to in vivo cellular phenotypes $[19,20]$. By applying micropatterning techniques to cell cultures, one can instead realize ECM proteins islands of different geometries and dimensions, specific for the chosen cellular type, thus controlling the cellular growth and behaviour.

Among the ECM proteins, the laminin class of glycoproteins are the most represented in the basement 
membranes, where it plays an important role in stimulating the growth and the differentiation of various cell types, supporting the cellular adhesion and inducing the cellular polarity. Moreover, laminins strongly affect the morphogenesis of the nervous system and promote the neurite outgrowth. All these functions are mediated by specific interactions between the protein domains and the cell membrane receptors, primarily the integrins. In vitro studies of the effects of laminin on cell functions have been carried out by adsorbing the protein onto the surface of cell-culture plastics or glassware, thus realizing patterned [21] and unpatterned [20] artificial matrices. These works clearly demonstrated that the physiological properties of laminin matrices depend strongly on their morphology [22] and that cells cultured on patterned laminin exhibit a more physiological phenotype than cells on unpatterned substrates [19]. However, to date no study has been carried out on the morphology of LN-1 layers by high resolution scanning probes.

In this work, we report on self-assembled extracellular matrix protein networks realized by soft lithography, demonstrating the physiological polymerization of laminin-1 (LN-1). High-quality $\mathrm{LN}-1$ patterns were realized by $\mu \mathrm{CP}$ lithography and investigated by indirect immunofluorescence and atomic force microscopy (AFM), in order to assess both the well-retained antigenicity of the proteins, and their supramolecular structural organization. In particular, the cross-shaped quaternary structure of single $\mathrm{LN}-1$ molecules and their self-assembling in characteristic polygonal networks were observed, resulting in mesh-like protein matrices by physisorption onto glass without any previous surface functionalization.

\section{Materials and methods}

\subsection{Stamp fabrication}

Our master patterns were fabricated by photolithography on $\mathrm{Si} / \mathrm{SiO}_{2}\left(\mathrm{SiO}_{2}\right.$ thickness $\left.\cong 100 \mathrm{~nm}\right)$ substrates. After the deposition of standard photoresist (Clariant AZ5214E:AZ EBR Solvent 1:1), UV-exposure $\left(8^{\prime} 30^{\prime \prime}\right.$ at $45 \mathrm{~W}$ ) and developing, we etched the thermal dioxide by using $\mathrm{NH}_{4} \mathrm{~F} / \mathrm{HF} / \mathrm{H}_{2} \mathrm{O}(6.6 \mathrm{~g} / 1.6 \mathrm{ml} / 10 \mathrm{ml})$. The so obtained $\mathrm{SiO}_{2}$ mask was then employed to etch Silicon by $5.9 \mathrm{M}$ solutions of $\mathrm{KOH}$ at $80^{\circ} \mathrm{C}$. Elastomeric replicas of hard masters were realized by in situ polymerization of Poly(dimethylsiloxane) (PDMS) (Sylgard 184, A:B 1:9, Dow Corning) at $140^{\circ} \mathrm{C}$ for $15 \mathrm{~min}$.

\subsection{Surface cleaning}

To remove organic residues and to hydroxilate the surface, glass coverslips (\#1, Menzel-Glaser) were cleaned by immersion in a freshly prepared piranha solutions $\left(3: 1, \mathrm{H}_{2} \mathrm{SO}_{4}: \mathrm{H}_{2} \mathrm{O}_{2}\right)$ for $90 \mathrm{~min}$ and thoroughly rinsed three times for $5 \mathrm{~min}$ in bidistilled and deionized water under ultrasonication at room temperature.

\subsection{Imprinting procedure}

$\mu \mathrm{CP}$ was carried out according to the scheme of Fig. 1. $10 \mu \mathrm{g} / \mathrm{ml}$ and $30 \mu \mathrm{g} / \mathrm{ml}$ solutions of Laminin from Engelbreth-Holm-Swarm murine sarcoma (EHS, Sigma-Aldrich), LN-1, in phosphate buffer saline (PBS, $\mathrm{pH}=7.4$ at $25^{\circ} \mathrm{C}$ ) were deposited on the patterned side of PDMS molds for $30 \mathrm{~min}$. Then, the protein-coated elastomeric elements were dried by a nitrogen stream and placed under their own weight for $5 \mathrm{~min}$. After peeling-off the replicas, the patterned coverslips were vigorously rinsed several times by bidistilled and deionized water in order to remove the unattached molecules of LN-1 and the PBS salt residues.

\subsection{Immunofluorescence}

After the stamping process, some patterns were washed in PBS solutions for $5 \mathrm{~min}$ and incubated for $45 \mathrm{~min}$ in primary antibody (1:25 rabbit anti-laminin polyclonal antibody, Sigma-Aldrich). Then, the patterns were washed for $5 \mathrm{~min}$ with PBS solution and incubated for $30 \mathrm{~min}$ in secondary antibody (1:32 rhodaminelabelled goat anti-rabbit IgG antibody, Sigma-Aldrich). Patterns were further washed with PBS for $5 \mathrm{~min}$, rinsed with bidistilled and deionized water to remove the salt

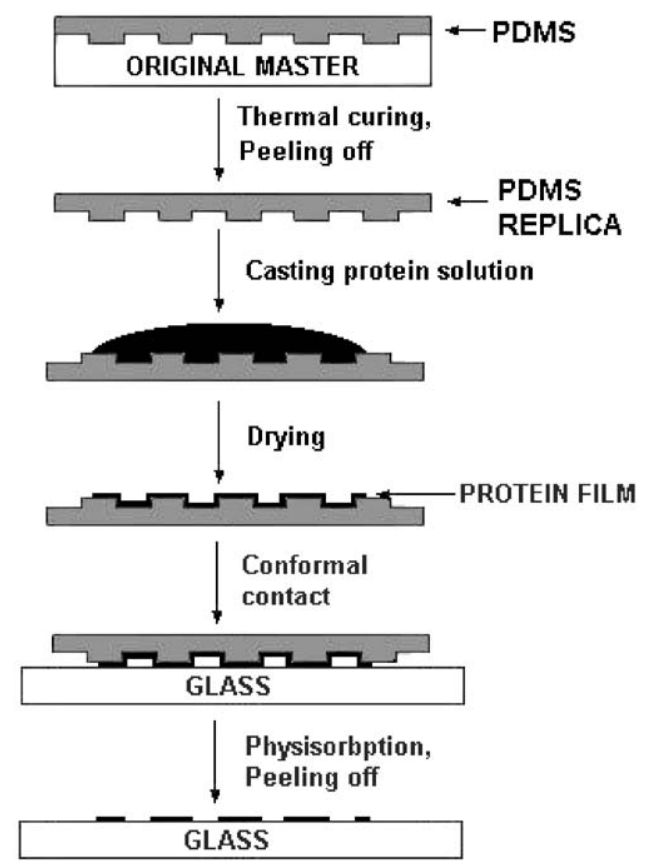

Fig. 1. Schematic diagram of the performed $\mu \mathrm{CP}$ process (features not in scale). 
residues, dried with nitrogen and photographed using a Leika fluorescence microscope.

\subsection{AFM characterization}

The supramolecular organization of $\mathrm{LN}-1$ within the patterned stripes was investigated by tapping-mode AFM in air using a Nanoscope III controller with a Bioscope microscope (Digital Instruments), integrated with a scanner having a maximum scan size of $90 \mu \mathrm{m}$. TESP-NCH Silicon probes (Veeco) were employed with a resonant frequency of about $320 \mathrm{kHz}$.

\section{Results and discussion}

We exploited the gentle process of $\mu \mathrm{CP}$ [7] to pattern regions of physisorbed $\mathrm{LN}-1$ onto glass substrates. $\mathrm{LN}-1$ is a large $(900 \mathrm{kDa})$ cruciform shaped heterotrimeric glycoprotein (Fig. 2), whose structure shows a $77 \mathrm{~nm}$ long arm, and three short arms, two of which $36 \pm 6 \mathrm{~nm}$ long and one $48 \pm 4 \mathrm{~nm}$ long [21,23,24]. Each of the $36 \mathrm{~nm}$-long arms contains a central and a terminal globular region separated by rod-like regions, whereas the $48 \mathrm{~nm}$ arm contains an additional globular region. The flexible rod-like long arm also ends with a large globular domain (Fig. 2). Such a structure is well suited for bridging between distant sites on cells and on others ECM proteins, so determining the cell-interactive role of $\mathrm{LN}-1$ in the basement membrane and in the nervous system. In addition, LN-1 is able to self-assembly, forming a network of polygonal structures [22,25,26]. This polymerization is not due to random protein aggregation. Instead it is a well-defined process, induced by the interaction between the terminal globular domains of the arms $[22,25,26]$. Calcium was found to be the most effective divalent cation mediating the

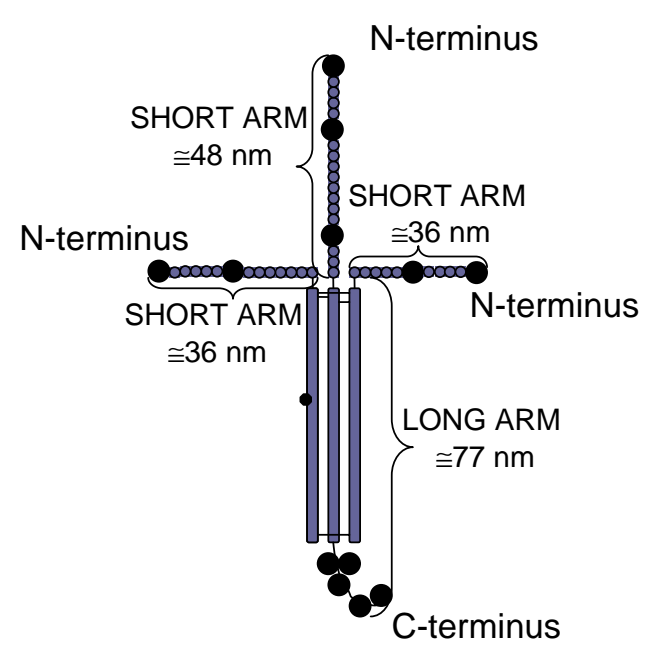

Fig. 2. Laminin-1 heterotrimer morphology. The globular domains of the protein are indicated by the black dots. self-assembling process [25]. The properties of in vitro laminin matrices strongly depend on their morphologies, similarly to what occurs in in vivo tissues [22]. In fact, Freire and coworkers demonstrated that when laminin, deposited onto glass coverslips, polimerized in a flat network with distinguishable regular polygons, the neuritogenesis and the promotion of the neurite outgrowth of embryonic neurons was more pronounced than on largely aggregated laminin matrices [22]. For this reason, we carefully investigated the effect of the imprinting process on the laminin supramolecular organization.

First, we performed indirect immunofluorescence assays on patterned substrates prepared by differently diluted $(10 \mu \mathrm{g} / \mathrm{ml}$ and $30 \mu \mathrm{g} / \mathrm{ml}) \mathrm{LN}-1$ solutions (Fig. 3), in order to check the retained antigenicity properties of our LN-1 patterns, and to assess the uniformity of the achieved patterns. We obtained similar results with the two concentrations, finding that the lithography process on glass results in well-defined features on the whole printed area (about $1 \mathrm{~cm}^{2}$ ) and that the epitopes of the LN-1 molecules are recognized from the anti-laminin antibody, which can indicate negligible conformational changes of the proteins. Therefore, a pattern of antigenic LN-1 molecules can be obtained by direct printing through protein physisorption on the substrate, and without substrate functionalization to covalently bind the protein. Such a result clearly contributes to the sample biocompatibility and has the advantage to be achieved by an easy and quick one-step lithographic process. We underline that the proteins strongly adhere to the substrate, since the long time of agitation, during

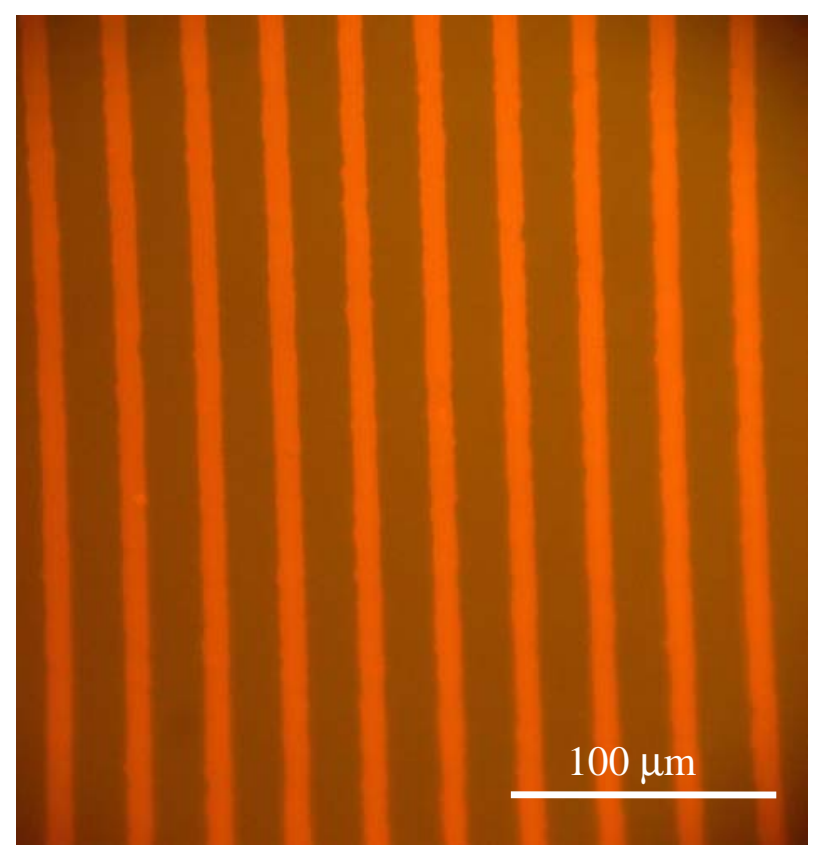

Fig. 3. Immunofluorescence micrograph of the obtained LN-1 pattern. The printed stripes are about $11 \mu \mathrm{m}$-wide. 
rinses and antibody incubations, does not cause the detachment of the LN-1 pattern from glass. The hydroxylation of glass induced by the piranha solution could certainly contribute to determine a strong interaction between $\mathrm{LN}-1$ and the coverslip surface, as confirmed by the poor patterns obtained by cleaning the glass with organic solvents only, showing large regions without adhered proteins.

Then, we investigated the inner structure of the printed laminin matrices by AFM on a sub- $\mu \mathrm{m}$ scale. In the case of the $10 \mu \mathrm{g} / \mathrm{ml}$ solution, we found evidence of the LN-1 self-assembling in its characteristic physiological polygonal network (Fig. 4), indicating that the arms terminal domains correctly interact with each other. As the formation of these structures is favoured
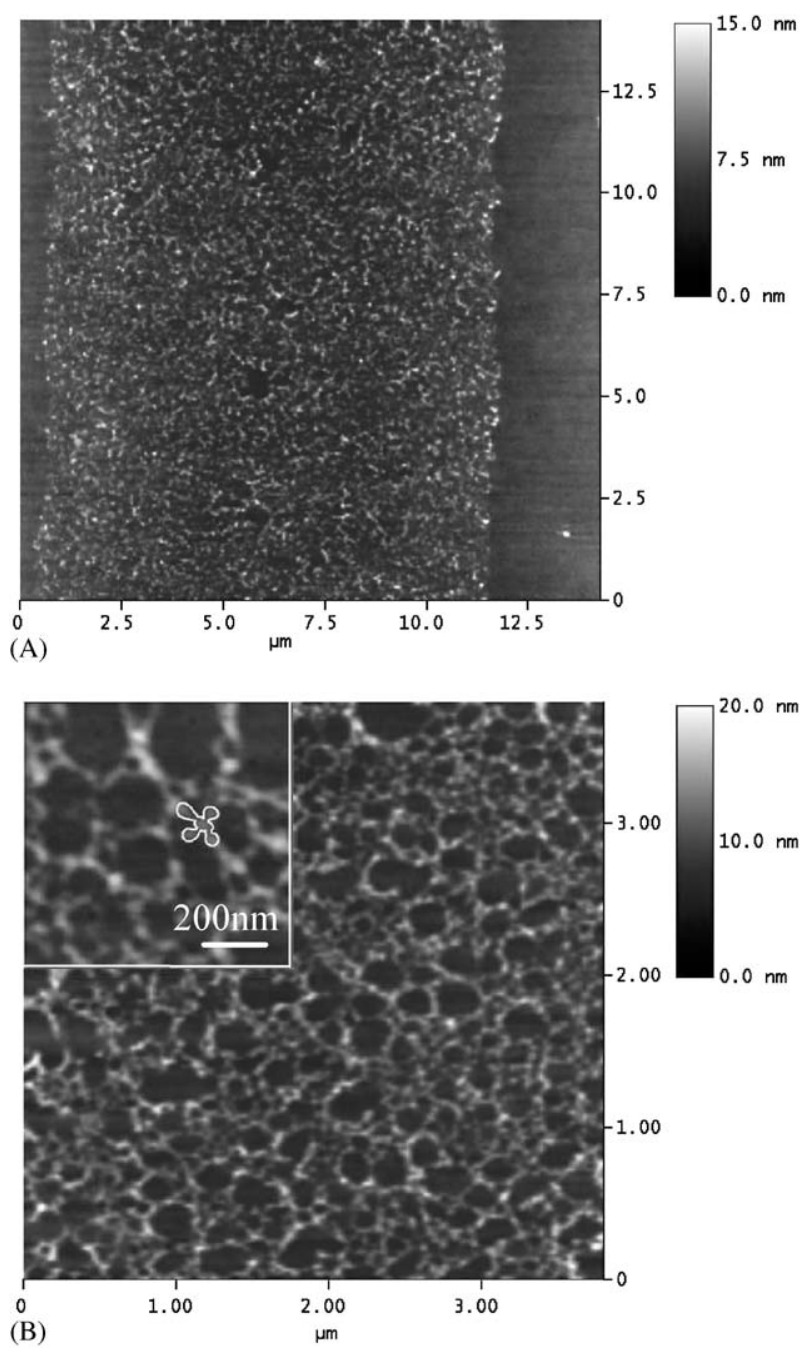

Fig. 4. (A) AFM image of a printed LN-1 stripe from $10 \mu \mathrm{g} / \mathrm{ml}$ PBS solutions. (B) AFM view of the self-assembled protein network within the printed region. The measured heights of the protein layer transferred to glass is comprised between 3 and $10 \mathrm{~nm}$. Inset: Higher resolution view, in which the single proteins are clearly appreciable. We marked the edge of a single LN-1 molecule by the white line. The measured lengths of the protein arms are in excellent agreement with data reported in literature $[21,23,24]$. by $\mathrm{Ca}^{2+}$ [25], we argued that Calcium was present as contaminant in the used PBS solution. The observed polygons exhibit size comprised between about 587 and $161 \mathrm{~nm}$. We observed also sub-100 nm voids, likely due to the assembling geometries of some laminin arms. Besides the quite large width of the printed stripe (11$\mu \mathrm{m})$, the sub $100-\mathrm{nm}$ underlying self-assembled LN-1 molecules are clearly visible in Fig. 4.

In addition, among several possible shapes of single LN-1 molecules observed in other studies [21], in which the flexible arms are involved in various bending and folding conformations, we identified the typical cruciform shape of LN-1 (Fig. 4B). This is favoured by the loss of the flexibility of the protein arms, which are blocked by their mutual interactions during the polimerization process. The characteristic dimensions of the LN-1 arms and the well-recognizable terminal globular domains are also seen in the inset of Fig. 4B.

Interestingly, the physiological organization of the physisorbed LN-1 network does not occur by employing more concentrated protein solutions $(30 \mu \mathrm{g} / \mathrm{ml})$. In fact, higher protein concentrations resulted in an apparently random laminin polymerization and in the impossibility to recognize single LN-1 molecules (Fig. 5), notwithstanding the uniformity of the pattern revealed by the indirect immunofluorescence assays. We believe that the observed dependence of the morphology on the concentration of the protein solution comes from the transfer mechanism by $\mu \mathrm{CP}$, depositing the threedimensional structure of the protein matrix, already formed in solution, onto the two-dimensional surface of the substrate. Indeed, high concentrations can determine a dense cross-linked three-dimensional matrix in solution, which could collapse during the drying of the replicas, and then be transferred to glass by imprinting, thus resulting in a multilayer of overlapped molecules. On the contrary, when the concentration of the protein

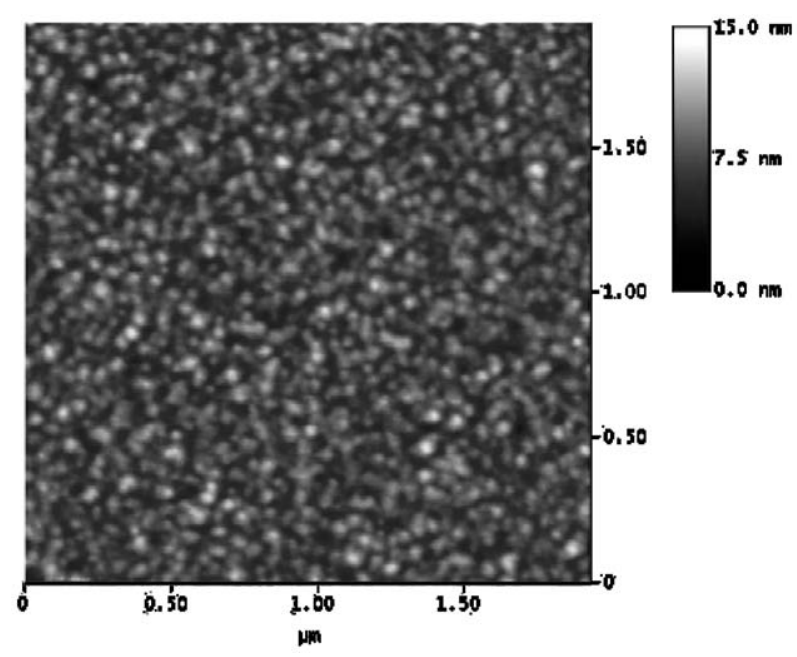

Fig. 5. AFM image of a LN-1 patterned region, printed from $30 \mu \mathrm{g} / \mathrm{ml}$ PBS solutions. 
solution is lower, the resulting matrix is less intricate, thus allowing one to transfer only a cross-linked monolayer of molecules onto glass. The dependence of the resulting $\mathrm{LN}-1$ supramolecular organization on the protein solution concentration is a crucial factor to be pointed out to realize ordered LN-1 monolayers by $\mu \mathrm{CP}$, which can be correctly recognizable by the cellular receptors. Further experimental studies are currently in progress in our laboratory to better investigate this point.

\section{Conclusion}

In conclusion, we demonstrated functional $\mathrm{LN}-1$ patterns on different scale-lengths. The $\mu \mathrm{m}$-size of the pattern features is determined by the $\mu \mathrm{CP}$ lithography and, more important, the protein self-assembling exhibits polygonal networks with clear sub-100 nm quaternary structures of laminin. The formation of these physiological mesh-like protein matrices by $\mu \mathrm{CP}$ technique was found to be strongly dependent on the LN-1 initial concentration, and it was achieved by physisorption without any previous chemical deposition onto the glass surfaces. This finding strongly candidates one-step soft lithography as a powerful technique to realize both micropatterned and physiologically selfassembled layers with biocompatible properties for cell culture applications in both basic biology studies and biosensors, drug discovery, and hybrid devices.

\section{References}

[1] Chen CS, Mrksich M, Huang S, Whitesides GM, Ingber DE. Geometric control of cell life and death. Science 1997;276:1425-8.

[2] Ito Y. Surface micropatterning to regulate cell functions. Biomaterials 1999;20:2333-42.

[3] Di Fabrizio E, Gentili M, Morales P, Pilloton R, Mela J, Cantucci S, Sese A. Microlithographic techniques for laser assisted fabrication of bioelectronic devices. Appl Phys Lett 1996;69:3280-2.

[4] Joachim C, Gimzewski J, Aviram A. Electronics using hybridmolecular and mono-molecular devices. Nature 2000;408:541-8.

[5] Rinaldi R, Biasco A, Maruccio G, Cingolani R, Alliata D, Andolfi L, Facci P, De Rienzo F, De Felice R, Molinari E. Solidstate molecular rectifier based on self-organized metalloproteins. Adv Mat 2002;14:1453-7.

[6] Martin AS, Sambles JR. Molecular rectifier. Phys Rev Lett 1993;70:218-21.

[7] Xia Y, Whitesides GM. Soft lithography. Angew Chem Int Ed 1998:37:550-75.
[8] Kane RS, Takayama S, Ostuni E, Ingber DE, Whitesides GM. Patterning proteins and cells using soft lithography. Biomaterials 1999;20:2363-76.

[9] Fang A, Ng H, Su X, Li SFY. Soft-lithography-mediated submicrometer patterning of self-assembled monolayer of hemoglobin on ITO surfaces. Langmuir 2000;16:5221-6.

[10] Delamarche E, Bernard A, Schmid H, Michel B, Biebuyck H. Patterned delivery of immunoglobulins to surfaces using microfluidic networks. Science 1997;276:779-81.

[11] Delamarche E, Bernard A, Schmid H, Bietsch A, Michel B, Biebuyck H. Microfluidic networks for chemical patterning of suctrate: design and application to bioassays. J Am Chem Soc 1998;120:500-8.

[12] Caelen I, Bernard A, Juncker D, Michel B, Heinzelmann H, Delamarche E. Formation of gradients of proteins on surfaces with microfluidic networks. Langmuir 2000;16:9125-30.

[13] Papra A, Bernard A, Juncker D, Larsen NB, Michel B, Delamarche E. Microfluidic networks made of poly(dimethylsiloxane), $\mathrm{Si}$, and $\mathrm{Au}$ coated with polyethylene glycol for patterning proteins onto surfaces. Langmuir 2001;17:4090-5.

[14] Bernard A, Delamarche E, Schmid H, Michel B, Bosshard HR, Biebuyck H. Printing patterns of proteins. Langmuir 1998;14: 2225-9.

[15] James CD, Davis RC, Kam L, Craighead HG, Isaacson M, Turner JN, Shain W. Patterned protein layers on solid substrates by thin stamp microcontact printing. Langmuir 1998;14:741-4.

[16] Bernard A, Renault JP, Michel B, Bosshard HR, Delamarche E. Microcontact printing of proteins. Adv Mat 2000;12:1067-70.

[17] Huang S, Ingber DE. The structural and mechanical complexity of cell-growth control. Nat Cell Biol 1999;1:E131-8.

[18] Boudreau N, Bissell MJ. Extracellular matrix signaling: integration of form and function in normal and malignant cells. Curr Opin Cell Biol 1998;10:640-6.

[19] Singhvi R, Kumar A, Lopez GP, Stephanopoulos GN, Wang DIC, Whitesides GM, Ingber DE. Engineering cell shape and function. Science 1994;264:696-8.

[20] Clark P, Britland S, Connolly P. Growth cone guidance and neuron morphology on micropatterned laminin surfaces. J Cell Sci 1993;105:203-12.

[21] Chen CH, Clegg DO, Hansma HG. Structures and dynamic motion of laminin-1 as observed by atomic force microscopy. Biochemistry 1998;37:8262-7.

[22] Freire E, Gomes FCA, Linden R, Moura NV, Coelho-Sampaio T. Structure of laminin substrate modulates cellular signaling for neuritogenesis. J Cell Sci 2002;115:4867-76.

[23] Engel J, Odermatt E, Engel A, Madri JA, Furthmayr H, Rohde H, Timpl R. Shapes, domain organization and flexibility of laminin and fibronectin, two multifunctional proteins of the extracellular matrix. J Mol Biol 1981;150:97-120.

[24] Bruch M, Landwehr R, Engel J. Dissection of laminin by cathepsin $G$ into its long arm and short arm structures and localization of regions involved in calcium dependent stabilization and self-association. Eur J Biochem 1989;185:271-9.

[25] Yurchenco PD, Cheng Y-S. Self-assembly and calcium-binding sites in laminin. J Biol Chem 1993;268:17286-99.

[26] Yurchenco PD, Tsilibary EF, Charonis A, Furthmayr H. Laminin polymerization in vitro. J Biol Chem 1985;260:7636-44. 